\title{
Intelligent Detector of Internal Combustion Engine Cylinder Pressure and Sensitivity Temperature Coefficient Compensation
}

\author{
Beirong Zheng, ${ }^{1}$ Chen Zhou, ${ }^{1}$ Xiaomin Pan, ${ }^{1}$ Quan Wang, ${ }^{2}$ and Wei Xue \\ ${ }^{1}$ College of Mechanical and Electrical Engineering, Wenzhou University, Wenzhou 323035, China \\ ${ }^{2}$ State Key Lab of Transducer Technology, Chinese Academy of Sciences, Shanghai 200050, China \\ Correspondence should be addressed to Wei Xue; xue_wenzhou@163.com
}

Received 10 July 2013; Accepted 1 September 2013

Academic Editor: Yan Yang

Copyright (c) 2013 Beirong Zheng et al. This is an open access article distributed under the Creative Commons Attribution License, which permits unrestricted use, distribution, and reproduction in any medium, provided the original work is properly cited.

\begin{abstract}
The detecting device based on mechanical mechanism is far from the measurement of internal combustion engine cylinder explosion and compression pressure. This pressure detection is under the environment of pulsed gas (over 500 times per one minute) and mechanical impactive vibration. Piezoresistive detection with silicon on insulator (SOI) strain gauges to pressure seems to be a good solution to meet such special applications. In this work, separation by implanted oxygen (SIMOX) wafer was used to fabricate the high temperature pressure sensor chip. For high accuracy and wide temperature range application, this paper also presents a novel pressure sensitivity temperature coefficient (TCS) compensation method, using integrated constant current network. A quantitative compensation formula is introduced in mathematics. During experiments, the absolute value of the compensated TCS is easy to be $10 \times 10^{-6} /{ }^{\circ} \mathrm{C} \sim 100 \times 10^{-6} /{ }^{\circ} \mathrm{C}$ by individual adjustment and calibration of each device's temperature compensation. Therefore, the feasibility and practicability of this technology are tested. Again, the disadvantages are discussed after the research of the experiment data and the improvement methods are also given in the designing period. This technology exhibits the great potential practical value of internal combustion engine cylinder pressure with volume manufacturing.
\end{abstract}

\section{Introduction}

Explosion pressure and compression pressure of internalcombustion engine cylinder are two significant technical parameters which affect its work $[1,2]$. Grasping its regularity of pressure is a main basis for designing and evaluating working performance of the internal-combustion engine. Currently, tools used to detect this pressure mainly are detection devices such as old mechanical watches. These mechanical detection watches are old-fashioned, which cause the low measuring accuracy (real measuring accuracy is far lower than marked accuracy). Besides, great swing of the clock pin makes it hard to read the number exactly and its service life is relatively short. It is far from meeting the operating requirement of internal-combustion engine cylinder's explosion pressure and compression pressure at the environment of striking by high temperature and pressure pulsed gas (over 500 times per one minute) and mechanical vibration.

There are three internal defects of mechanical pressure gauges $[3,4]$. They are mechanical hysteresis, stagnation or creeping, and elastic effect. Elastic effect certainly causes performance deviation. Besides, elastic modulus and rigidity modulus of elastic material are low, which leads to a bad dynamic response and affects its use severely [5]. The device presented in this paper equipped with amplified circuit and eight characters LCD screen avoids the detection device and utilizes high temperature piezoresistive pressure sensors as detecting units. The sensor makes it convenient to operate and has high testing precision and more scientific results. At the same time, it possesses several functions of data detection and record.

\section{Fabrication of High Temperature Piezoresistive Pressure Sensor}

Silicon pressure sensors were the first micromechanical devices developed. Using piezoresistive effect and microelectronic technology of semiconductor silicon, they have many advantages: large output signal, high SNR (signal-to-noise), convenient circuit processing, and high frequency responses [6]. 
The conventional piezoresistive pressure sensor chip functions as a Wheatstone bridge, formed by four diffused or ion implanted strain gauges in the thin silicon diaphragm. Silicon exhibits a high gauge factor and is principally applicable as a piezoresistive material up to temperatures of $400^{\circ} \mathrm{C}$ [7]. However, a known limitation of these silicon-based devices using isolation by reverse biased pn-junctions is the rising junction leakage current at elevated temperatures. The promising attempts for fabrication of high temperature piezoresistive pressure sensors is to cancel pn-insulation of the piezoresistors. One of the basic ideas is silicon on insulator (SOI) technology to remedy this by electrically insulating the thin layer at the wafer surface carrying the electronic device from the bulk wafer used as mechanical support $[8,9]$.

Two mainstream technologies of manufacturing SOI wafer are separation by implantation of oxygen $[10,11]$ (SIMOX) and bonding technology. SIMOX technology refers to the technology where large dose oxygen ions are implanted into initial silicon, then SOI structure is formed after high temperature annealing. Bonding technology includes bonding and etch back SOI technology and Smart Cut (or UNIBON) technology $[12,13]$. This paper adopted SIMOX technology, implanting oxygen ions into n-type silicon to obtain silicon dioxide dielectric isolated SOI wafer with high quality and commercial use. Then silicon chip is manufactured on micro fabrication platform. Since buried layer is isolated by silicon dioxide and pn-junction isolation is cancelled, the leakage current caused by temperature increasing between upper silicon measurement unit and bulk silicon is avoided. The sensor packaged with this kind of sensing chip is presented with high accuracy and a good long-term stability in high temperature testing experiments.

Figure 1 is the high temperature pressure sensor gauge chip based on SIMOX SOI wafer. One circular shape diaphragm is taken as the sensitive structure layer. The chip still retains silicon as the sensing material but has silicon oxide as an electrically isolating layer between it and the substrate. The sensor packaged with this kind of sensing chip is presented with high accuracy and a good longterm stability in high temperature testing experiments. The designed chip film thickness is $0.04 \mathrm{~mm}$ and effective radius of diaphragm is $2 \mathrm{~mm}$.

When the sensor is subjected to pressure, the gas (or liquid) with high temperature and high pressure acts on the back of the chip and causes it to be deformed. The deformation is converted into the resistance variation in the gauges of the Wheatstone bridge on the other side. The Wheatstone bridge transforms the related stress, due to pressure, into an electrical output. Then this pressure can be measured. The diaphragm of the sensor can be optimized to make sensors of different ranges.

\section{Testing of Dynamic Characteristics}

The natural frequency of peripheral clamped circular diaphragm $[14] f_{n}$ is

$$
f_{n}=\frac{2.54 h}{\pi r^{2}} \sqrt{\frac{E}{3\left(1-v^{2}\right) \rho}},
$$

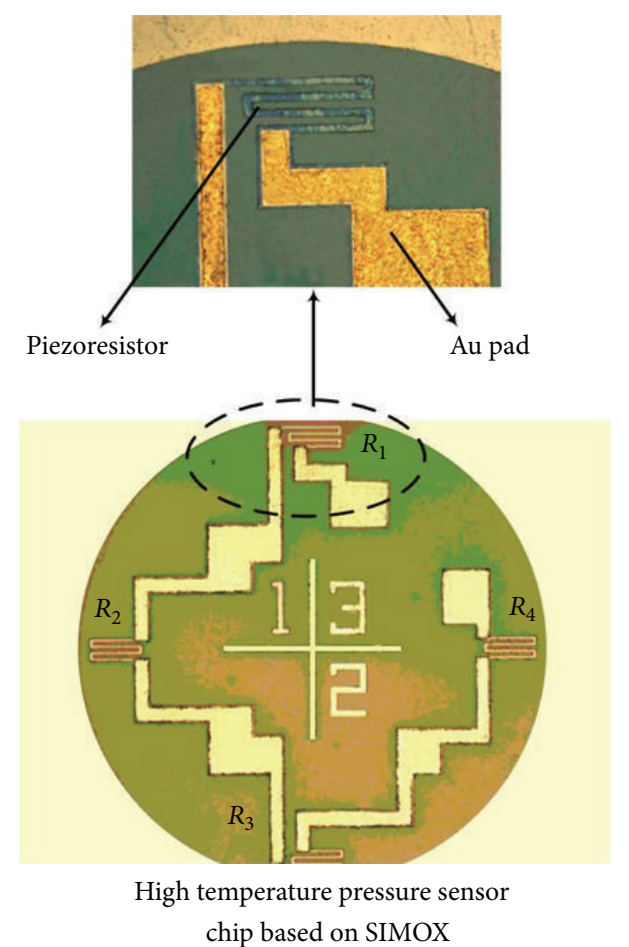

FIGURE 1: High temperature sensor chip based on SIMOX was used for strain gauges in detecting internal-combustion engine cylinder pressure.

where $E$ is young modulus, $v$ is Poisson's ratio, $\rho$ is material density, and $r$ is the effective radius of diaphragm.

Young's modulus of silicon is almost equal to steel material, but its density is only between $1 / 3$ and $1 / 4$ density of steel. So natural frequency of silicon film is higher than steel sheets by two times. The designed film thickness is $0.04 \mathrm{~mm}$, and effective radius of diaphragm is $2 \mathrm{~mm}$. It is easy to know that theoretic natural frequency is $44.22 \mathrm{kHz}$. It compared well with dynamic calibrated frequency of shock tube which is $38.46 \mathrm{kHz}$. It is shown in Figure 2 that it meets the requirement of high frequency measurement.

\section{TCS Compensation}

Piezoresistive pressure sensor is sensitive to the ambient temperature change. The temperature dependence of sheet resistance is one of the main limiting factors to improve the accuracy of the device and extend its application $[15,16]$.

Although various types of piezoresistive pressure sensors are available, for all integrated solid-state piezoresistive sensors, the basic operating principle of the sensor element remains unchanged. A pressure is applied to the resistors made with semiconductor materials in the arms of the bridge change by some amount. These devices use diffused or ion implanted resistors in an integral silicon diaphragm to transform the related stress, due to pressure, into an electrical output. 


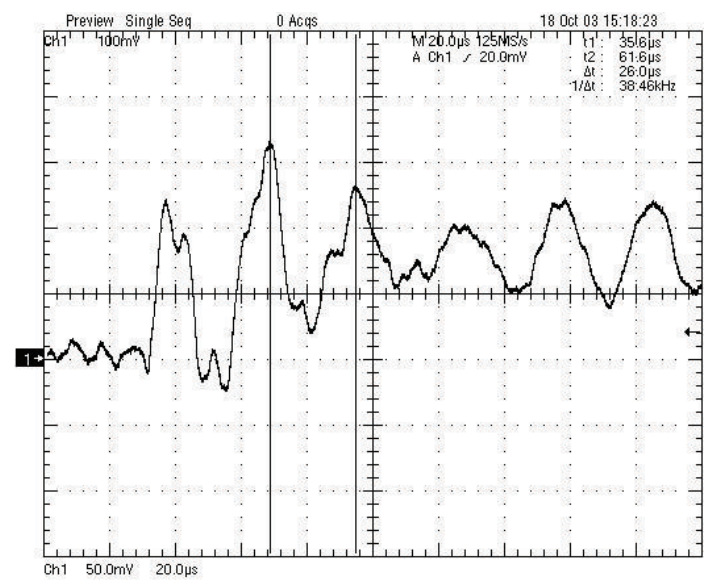

Figure 2: Dynamic response of the intelligent detector with the SIMOX silicon chip to meet mechanical impactive vibration.

They generally function as a Wheatstone bridge. That is, $R_{1}=R_{2}=R_{3}=R_{4}, \Delta R_{1}=\Delta R_{3}=-\Delta R_{2}=-\Delta R_{4}$. So, the output voltage, $V_{0}$, is easily shown to be

$$
V_{0}=V_{B} \cdot \frac{\Delta R}{R} .
$$

Since the change in resistance is directly proportional to pressure, $V_{0}$ can be written as

$$
V_{0}=S \cdot P \cdot V_{B} \pm V_{\mathrm{OS}},
$$

where $V_{0}$ is the output in $\mathrm{mV}, S$ is the sensitivity in $\mathrm{mV} / \mathrm{V} / \mathrm{Pa}$, $P$ is the pressure in $\mathrm{Pa}, V_{B}$ is the bridge voltage in volts, and $V_{\mathrm{OS}}$ is the offset error (the differential output voltage when the applied pressure is zero). The offset voltage presents little problems in application since it can easily be compensated in the amplifier circuit or corrected digitally if a microprocessor is used in the system.

Looking at the derivative of (2) with respect to temperature and ignoring the $V_{\mathrm{OS}}$ term, we get

$$
V_{0}^{\prime}=P \cdot\left(S \cdot V_{B}^{\prime}+S^{\prime} \cdot V_{B}\right) \text {. }
$$

When a sensor is properly compensated, the output voltage does not change with the temperature.

Hence,

$$
V_{0}^{\prime}=0, \quad S \cdot V_{B}^{\prime}=-S^{\prime} \cdot V_{B}, \quad \text { or } V_{B}^{\prime} / V_{B}=-S^{\prime} / S .
$$

$S^{\prime} / S$ is the temperature coefficient of sensitivity (TCS). TCS is always below zero due to the decrease in piezoresistive coefficient with increasing temperature. In order to compensate for the change in sensitivity, the bridge voltage must change with equal magnitude in the opposite direction, that is, using positive temperature coefficient of bridge voltage compensates negative TCS.

Although the basic piezoresistive pressure sensor elements are often referred to as either constant current or constant voltage driven sensors, the two basic methods of

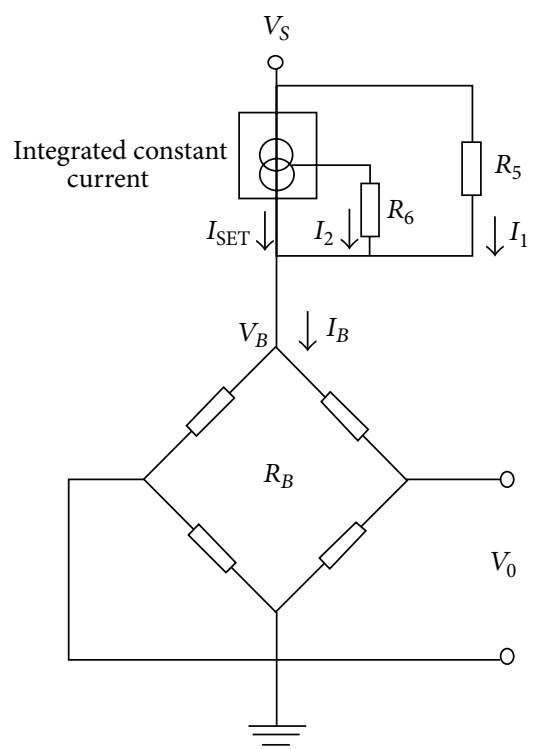

FIGURE 3: Sensitivity temperature coefficient compensation-based integrated constant current.

TCS compensation are using the internal characteristics of the die in conjunction with a constant source or using thermistors or other similar devices in conjunction with a constant voltage source $[17,18]$.

Thermistors can be used for TCS compensation. But they are inherently nonlinear, difficult to use in volume production, and more expensive than circuit approaches. The circuit is designed to incorporate a minimum number of adjustments and allow interchangeability of devices with little variation from device to device.

In this work, LM334 used for TCS compensation easily and inexpensively is shown in Figure 3. The LM334 is a three-pin programmable current source featuring an output current that rises linearly with temperature at $+3360 \% /{ }^{\circ} \mathrm{C}$. In Figure 3 , it is shown that

$$
V_{B}=I_{B} R_{B}=\left(I_{\mathrm{SET}}+I_{2}\right) R_{B}=\left(I_{\mathrm{SET}}+\frac{V_{S}-V_{B}}{R_{2}}\right) R_{B},
$$

Where $V_{B}$ is the Wheatstone bridge voltage, $I_{B}$ is Wheatstone bridge current, $R_{B}$ is Wheatstone bridge internal resistance $I_{\mathrm{SET}}$ is integrated constant current, $I_{1}$ and $I_{2}$ are the currents of the outer $R_{1}$ and $R_{2}$, and $V_{S}$ is the supply voltage.

Solving (6), it becomes

$$
V_{B}=\left(I_{\mathrm{SET}} R_{2}+V_{S}\right) \frac{R_{B}}{R_{B}+R_{2}} .
$$

As $\alpha=R_{B} /\left(R_{B}+R_{2}\right)$, taking the derivative with respect to temperature of (9) and normalizing it with respect to the bridge voltage, we obtain

$$
\frac{V_{B}^{\prime}}{V_{B}}=\left(\frac{R_{B}^{\prime}}{R_{\mathrm{B}}}\right)(1-\alpha)+\left(\frac{I_{\mathrm{SET}}^{\prime}}{I_{\mathrm{SET}}}\right)\left(1-\alpha\left(\frac{V_{S}}{V_{B}}\right)\right) .
$$


TABLE 1: TCS compensation measurement results.

\begin{tabular}{|c|c|c|c|c|c|c|c|}
\hline$V_{S}(\mathrm{~V})$ & $V_{B}(\mathrm{~V})$ & Times & No. & $\begin{array}{c}\text { Uncompensated } \\
\text { TCS } / \times 10^{-6} /{ }^{\circ} \mathrm{C}\end{array}$ & $R_{5}(\mathrm{~K} \Omega)$ & $R_{6}(\mathrm{~K} \Omega)$ & $\begin{array}{l}\text { Compensated } \\
\mathrm{TCS} / \times 10^{-6} /{ }^{\circ} \mathrm{C}\end{array}$ \\
\hline 6 & 4 & 1 & 1 & -2529 & 82 & 9.1 & 21 \\
\hline 6 & 4 & 2 & 1 & -2529 & 82 & 9.1 & 32 \\
\hline 6 & 4 & $3^{*}$ & 1 & -2529 & 82 & 9.1 & 25 \\
\hline 9 & 6 & 1 & 1 & -2529 & 56 & 8.2 & 48 \\
\hline 9 & 6 & 2 & 1 & -2529 & 56 & 8.2 & 30 \\
\hline 9 & 6 & $3^{*}$ & 1 & -2529 & 56 & 8.2 & 35 \\
\hline 12 & 9 & 1 & 1 & -2529 & 36 & 7.5 & -27 \\
\hline 12 & 9 & 2 & 1 & -2529 & 36 & 7.5 & -14 \\
\hline 12 & 9 & $3^{*}$ & 1 & -2529 & 36 & 7.5 & -20 \\
\hline 6 & 4 & 1 & 2 & -2617 & 75 & 9.1 & -19 \\
\hline 6 & 4 & 2 & 2 & -2617 & 75 & 9.1 & -26 \\
\hline 6 & 4 & $3^{*}$ & 2 & -2617 & 75 & 9.1 & -37 \\
\hline 9 & 6 & 1 & 2 & -2617 & 56 & 10 & 45 \\
\hline 9 & 6 & 2 & 2 & -2617 & 56 & 10 & 36 \\
\hline 9 & 6 & $3^{*}$ & 2 & -2617 & 56 & 10 & 39 \\
\hline 12 & 9 & 1 & 2 & -2617 & 33 & 7.5 & -20 \\
\hline 12 & 9 & 2 & 2 & -2617 & 33 & 7.5 & -38 \\
\hline 12 & 9 & $3^{*}$ & 2 & -2617 & 33 & 7.5 & -34 \\
\hline
\end{tabular}

Note: $3^{*}$ is referred to the third measurement data of TCS after compensation of TCO.

Solving the equation for resistor $R_{2}$, we get

$$
R_{2}=\frac{R_{B}\left[I_{\mathrm{SET}}^{\prime} / I_{\mathrm{SET}}\left(\left(V_{S} / V_{B}\right)-1\right)+V_{B}^{\prime} / V_{B}\right]}{\left(R_{B}^{\prime} / R_{B}\right)+\left(I_{\mathrm{SET}}^{\prime} / I_{\mathrm{SET}}\right)-\left(V_{B}^{\prime} / V_{B}\right)} .
$$

Substituting (7) into (9), we find that

$$
R_{2}=\frac{R_{B}\left[\left(I_{\mathrm{SET}}^{\prime} / I_{\mathrm{SET}}\right)\left(\left(V_{S} / V_{B}\right)-1\right)-\left(S^{\prime} / S\right)\right]}{\left(R_{B}^{\prime} / R_{B}\right)+\left(I_{\mathrm{SET}}^{\prime} / I_{\mathrm{SET}}\right)+\left(S^{\prime} / S\right)} .
$$

According to the three-pin programmable current source $I_{\text {SET }}=0.0677 / R_{1}$, once $R_{2}$ is known, $R_{1}$ can be derived from the following equation:

$$
R_{1}=\frac{0.0677 R_{B} R_{2}}{V_{B}\left(R_{B}+R_{2}\right)-V_{S} R_{B}} .
$$

To a senor chip, if the $V_{S}$ and $V_{B}$ are assumed, $R_{B}, S^{\prime} / S$, and $R_{B}^{\prime} / R_{B}$ can be obtained. With $I_{\mathrm{SET}}^{\prime} / I_{\mathrm{SET}}=3360 \times 10^{-6} /{ }^{\circ} \mathrm{C}$, substituting the above values into (10) and (11), $R_{1}$ and $R_{2}$ can be obtained.

In experiments, 10 pieces of sensor chips are picked up randomly. Initially, before the compensation, the calibration is done with the temperature characteristic of the sensor (after ageing). The compensating value of TCS can be calculated from the data of calibration. The absolute value of the compensated TCS is easy to be $10 \times 10^{-6} /{ }^{\circ} \mathrm{C} \sim 100 \times 10^{-6} /{ }^{\circ} \mathrm{C}$ by calibration of several temperature compensation cycles. Three experimental results of the ten sensors are listed in Table 1. Then the compensation of TCO (temperature compensation of offset) is measured [19]. The experiments show that if the basic feature of the sensor is stable, the absolute value of the compensated TCO is easy to be controlled in $10 \times 10^{-6} /{ }^{\circ} \mathrm{C} \sim 100 \times 10^{-6} /{ }^{\circ} \mathrm{C}$. Meanwhile, it shows that the changes are not so obvious for measuring the overall TCS of the sensor once again, as referred to the third group data in Table 1 . The feasibility of this method is subsequently demonstrated by the static calibrations and offset stability measurements, and then is promoted in line product of micropressure sensor.

For a pressure sensor, zero point stability for a long term is also an important parameter. The sensor zero point output under the ambient environment for 12 hours was measured. The output voltages are measured with recording the ambient temperature. Figure 4 shows the variation of output voltage. The pressure sensor has a strong anti-interference ability and can meet the requirement of high accuracy tests.

The intelligent detector of internal-combustion engine cylinder pressure is shown in Figure 5. It was packaged with high temperature piezoresistive pressure sensor to get the high frequency response as well as to perform under harsh environment. Equipped with preamplifier circuit, it was convenient to read the testing results, and the operating performance is reliable.

\section{Discussion}

Detectors were sent to Dalian Locomotive Works to carry contrast test with mechanical watch on DF4 locomotives testbed. The results presented that maximum power of the diesel engine is $2650 \mathrm{KW}$. Explosive pressure is over 


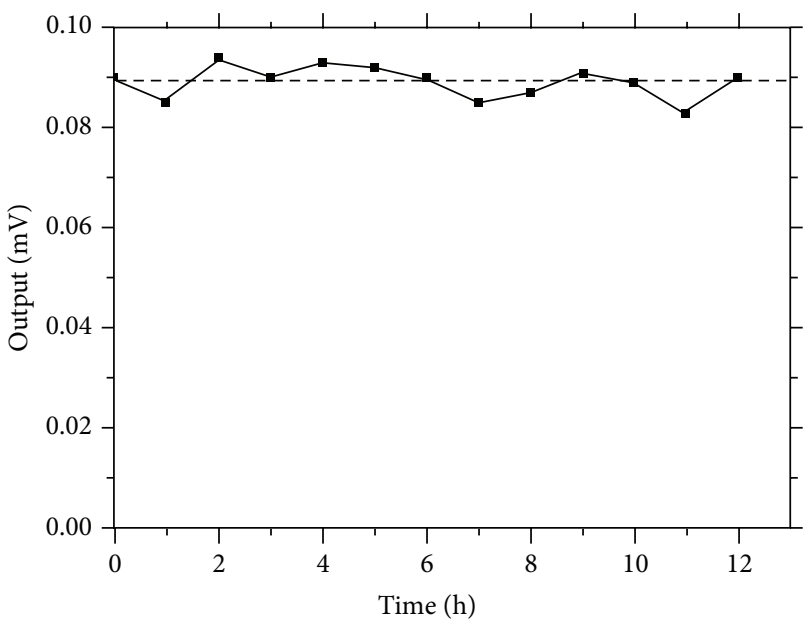

FIGURE 4: Intelligent detector's zero point stability under room temperature.

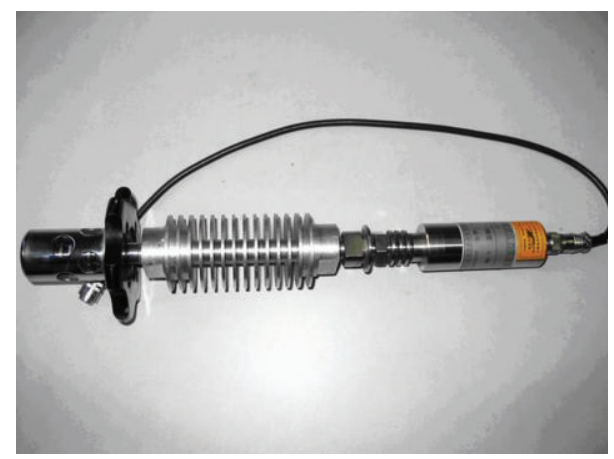

FIGURE 5: Intelligent detector of internal-combustion engine cylinder pressure with sensitivity temperature coefficient compensationbased integrated constant current.

12.55 MPa, and allowable error of air cylinders is $0.589 \mathrm{MPa}$. Contrast data of sixteen air cylinders is shown in Table 2.

Pressure recorded by detector was higher than testing result of mechanical watch. Mechanical watch produced major pressure loss during measurement process which was caused by its structure defect.

The head of mechanical watch consists of flattened and bent metal tube. The head produced deformation at the environment of high temperature and impure gas. There is a check valve of plane type inside. Its sealing is easy to be damaged by carbide in air, which will contribute to a much shorter life than detector. Sometimes mechanical watch may break down before finishing the test.

\section{Conclusion}

Intelligent detector of internal-combustion engine cylinder pressure with sensitivity temperature coefficient compensation-based integrated constant current is demonstrated in this paper. Separation by implanted oxygen (SIMOX) wafer was used to fabricate the high temperature pressure sensor chip. For high accuracy and wide temperature range
TABLE 2: Contrast testing data of the detector and the mechanical watch.

\begin{tabular}{lcc}
\hline $\begin{array}{l}\text { Cylinder } \\
\text { number }\end{array}$ & $\begin{array}{c}\text { Pressure value of } \\
\text { mechanical watch (MPa) }\end{array}$ & $\begin{array}{c}\text { Pressure value of the } \\
\text { detector (MPa) }\end{array}$ \\
\hline 1 & 11.80 & 12.60 \\
2 & 12.00 & 13.50 \\
3 & 11.80 & 13.15 \\
4 & 12.00 & 13.55 \\
5 & 11.80 & 13.20 \\
6 & 12.00 & 13.55 \\
7 & 11.80 & 12.85 \\
8 & 12.00 & 13.75 \\
9 & 12.10 & 13.05 \\
10 & 12.00 & 13.40 \\
11 & 12.00 & 13.85 \\
12 & 12.20 & 13.85 \\
13 & 12.20 & 13.50 \\
14 & 12.00 & 13.75 \\
15 & 12.20 & 13.30 \\
16 & 12.30 & 14.00 \\
\hline
\end{tabular}

application, this paper also presents a novel pressure sensitivity temperature coefficient (TCS) compensation method, using integrated constant current network. A quantitative compensation formula is introduced in mathematics. During experiments, the absolute value of the compensated TCS is easy to be $10 \times 10^{-6} /{ }^{\circ} \mathrm{C} \sim 100 \times 10^{-6} /{ }^{\circ} \mathrm{C}$ by individual adjustment and calibration of each device's temperature compensation. Intelligent detector effectively avoided the effect caused by high temperature and pressure pulsed gas, using high temperature piezoresistive pressure transducer. Meanwhile, the detector equipped with preamplifier circuit makes it convenient for the operator to read the results and guarantees the scientific and reasonable testing result.

\section{Acknowledgments}

This work was financially supported by the science and technology development plan of Zhejiang province (public technology research and industrial projects, Grant no. 2012C21088), natural science foundation of Zhejiang province (Grant no. LY13E050016), and the opening foundation of Jiangsu province material tribology key laboratory (no. kjsmcx1001).

\section{References}

[1] S. Satoshi, O. Yasuhiro, M. Tetsuo et al., "Instantaneous 2D imaging of temperature in an engine cylinder with flame combustion," International Journal of Heat and Mass Transfer, vol. 62, pp. 382-390, 2013.

[2] K. Halit, "Dynamic model of a two-cylinder four-stroke internal combustion engine and vibration treatment," International Journal of Engine Research, vol. 13, no. 6, pp. 616-627, 2012. 
[3] J. C. F. Millett, N. K. Bourne, M. Q. Chu, I. P. Jones, G. T. Gray III, and G. Appleby-Thomas, "The role of aging on the mechanical and microstructural response of aluminum 6061 to one-dimensional shock loading," Journal of Applied Physics, vol. 108, no. 7, Article ID 073502, 2010.

[4] M. Cabrini, S. Lorenzi, P. Marcassoli, and T. Pastore, "NN-SCC assessment of steels for buried pipelines by means of 3 point bending tests," Metallurgia Italiana, vol. 102, no. 3, pp. 5-11, 2010.

[5] L. Haifeng and N. Jianguo, "Mechanical behavior of reinforced concrete subjected to impact loading," Mechanics of Materials, vol. 41, no. 12, pp. 1298-1308, 2009.

[6] M. H. Bao, Micro Mechanical Transducers-Pressure Sensors, Accelerometers and Gyroscopes, Elsevier, Amsterdam, The Netherlands, 2000.

[7] A. Plŏßl and G. Krauter, "Silicon on insulator: material aspects and application," Solid-State Electronics, vol. 44, no. 5, pp. 775$782,2000$.

[8] G. K. Celler and S. Cristoloveanu, "Frontiers of silicon-oninsulator," Journal of Applied Physics, vol. 93, no. 9, pp. 49554978, 2003.

[9] A. Diab, I. Ionica, G. Ghibaudo et al., "RC model for frequency dependence of split C-V measurements on bare SOI wafers," IEEE Electron Device Letters, vol. 34, no. 6, pp. 792-794, 2013.

[10] Q. Wang, J. Ding, W. Xue, and Z. Ling, "Micro machining process of high temperature pressure sensor gauge chip based on SIMOX SOI wafer," International Journal of Materials and Product Technology, vol. 31, no. 2-4, pp. 375-385, 2008.

[11] A. F. Saavedra, K. S. Jones, M. E. Law, and K. K. Chan, "Comparison of 311 Defect Evolution in SIMOX and Bonded SOI Materials," Journal of the Electrochemical Society, vol. 151, no. 4, pp. G266-G270, 2004.

[12] H. Moriceau, F. Fournel, B. Aspar et al., "New layer transfers obtained by the SmartCut Process," Journal of Electronic Materials, vol. 32, no. 8, pp. 829-835, 2003.

[13] A. F. Saavedra, J. Frazer, K. S. Jones et al., "Influence of the surface Si/buried oxide interface on extended defect evolution in silicon-on-insulator scaled to $300 \AA$," Journal of Vacuum Science and Technology B, vol. 20, no. 6, pp. 2243-2247, 2002.

[14] D. F. Liu, Mechanical Sensitive Device and Its Application, Science Press, Beijing, China, 1987.

[15] J. Scott and E. T. Enikov, "Novel temperature compensation technique for force-sensing piezoresistive devices," Journal of Micromechanics and Microengineering, vol. 21, no. 11, Article ID 115017, 2011.

[16] N. P. Futane, S. R. Chowdhury, C. R. Chowdhury, and H. Saha, "ANN based CMOS ASIC design for improved temperaturedrift compensation of piezoresistive micro-machined high resolution pressure sensor," Microelectronics Reliability, vol. 50, no. 2, pp. 282-291, 2010.

[17] Q. Wang, J. Ding, and W. Wang, "Fabrication and temperature coefficient compensation technology of low cost high temperature pressure sensor," Sensors and Actuators A, vol. 120, no. 2, pp. 468-473, 2005.

[18] P. Melvås and G. Stemme, "A diode-based two-wire solution for temperature-compensated piezoresistive pressure sensors," IEEE Transactions on Electron Devices, vol. 50, no. 2, pp. 503509, 2003.

[19] S. Vlassis and S. Siskos, "Signal conditioning circuit for piezoresistive pressure sensors with variable pulse-rate output," Analog Integrated Circuits and Signal Processing, vol. 23, no. 2, pp. 153162,2000 . 

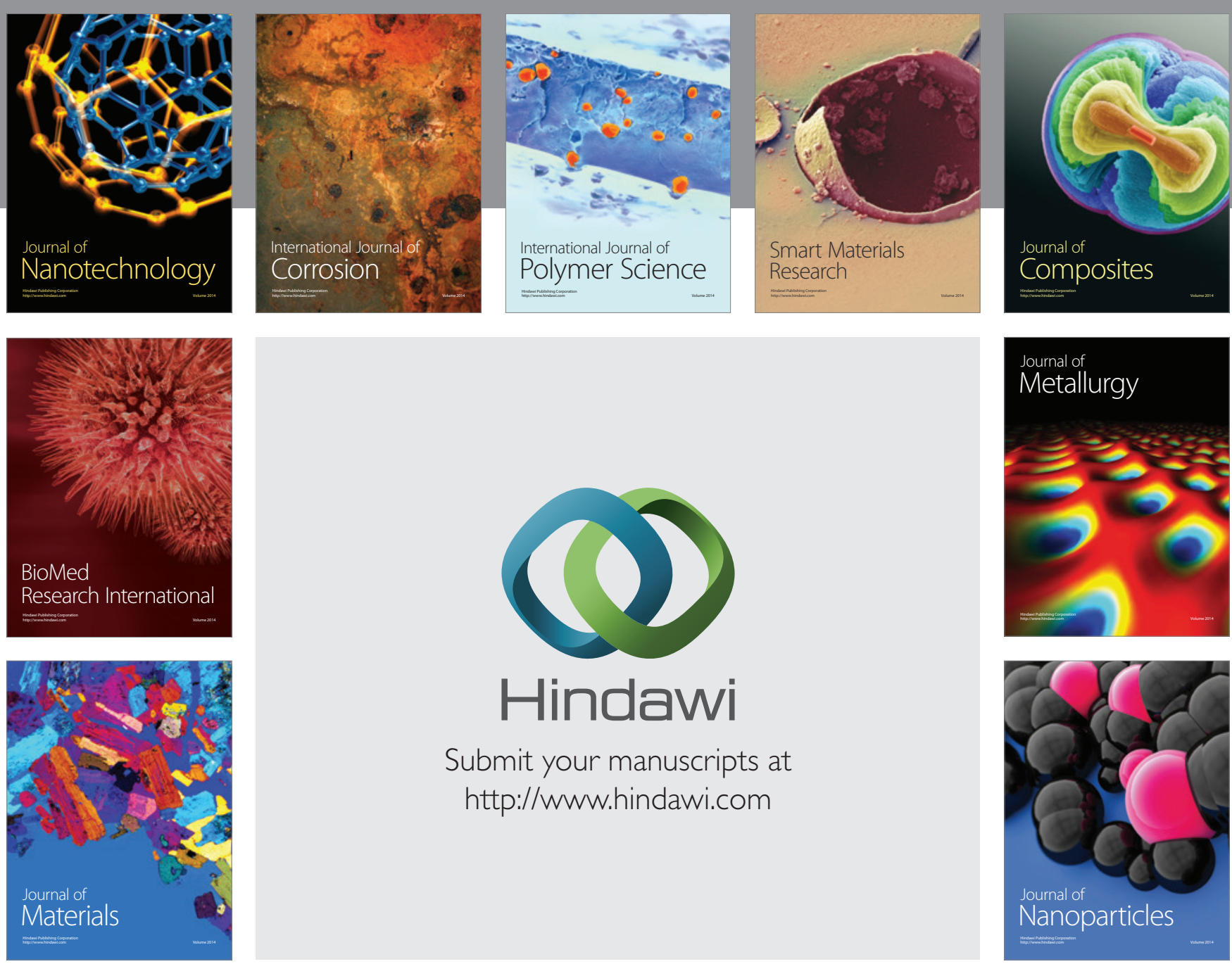

Submit your manuscripts at http://www.hindawi.com
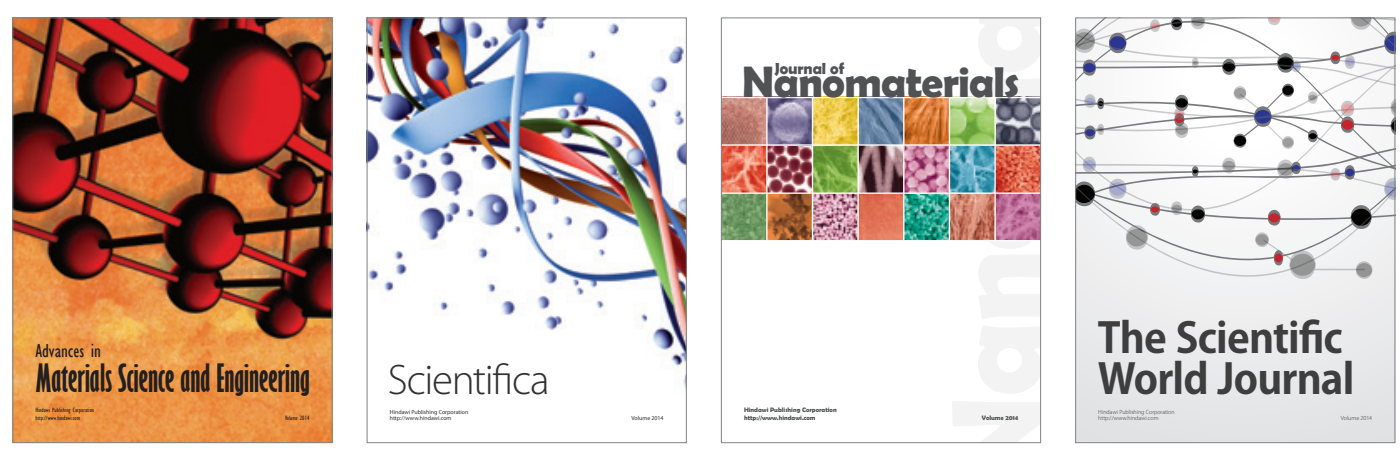

\section{The Scientific World Journal}
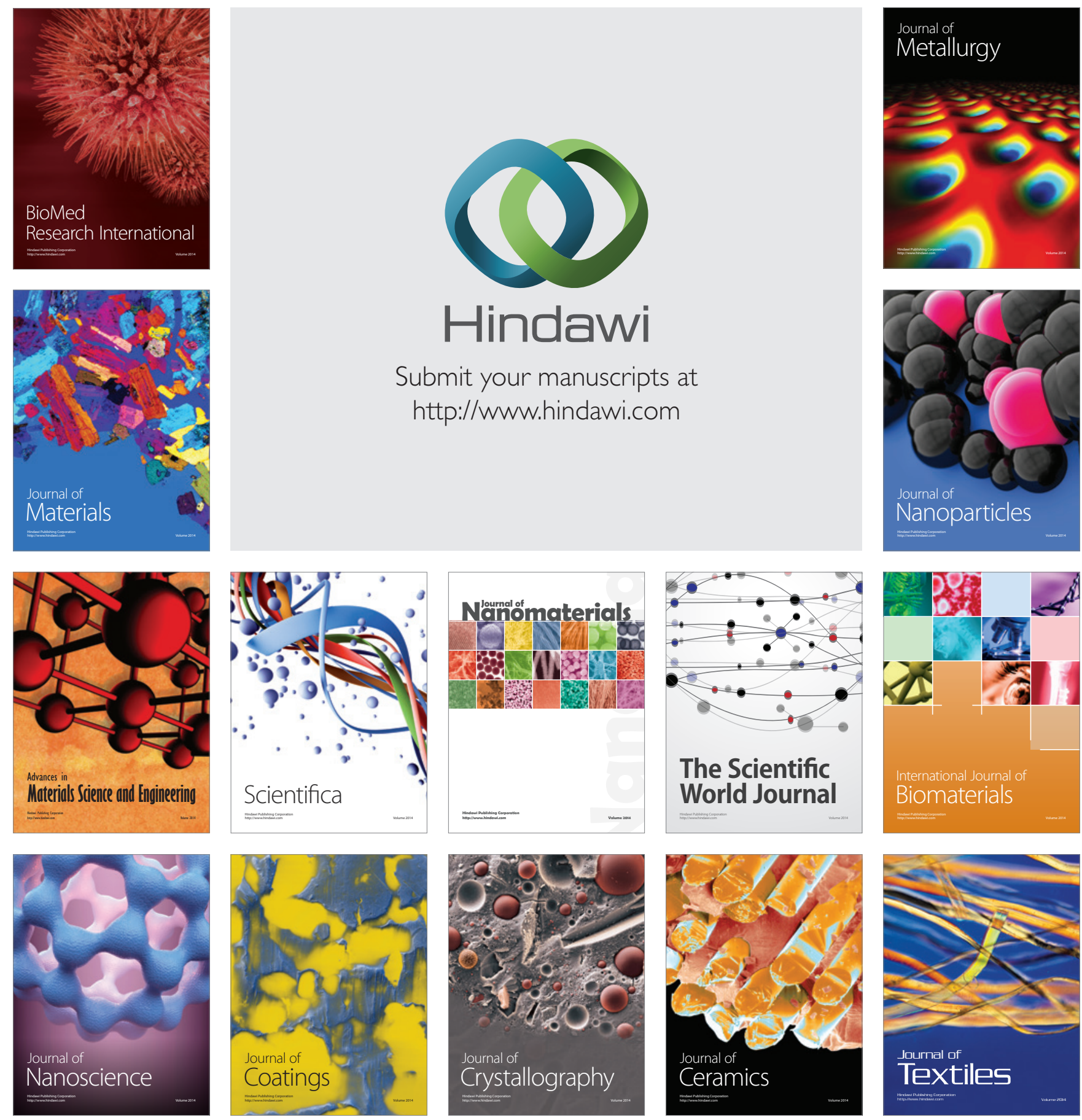